\title{
Estimation of the Randomness of Heart Rhythm Using Fractal Characteristics
}

\author{
Abdullaev Namik Tahir, Ibrahimova Irada Javad \\ Azerbaijan Technical University (Baku), Baku, Azerbaijan \\ Email: a.namik46@mail.ru,irada432@gmail.com
}

How to cite this paper: Tahir, A.N. and Javad, I.I. (2020) Estimation of the Randomness of Heart Rhythm Using Fractal Characteristics. Open Access Library Journal, 7: e6444.

https://doi.org/10.4236/oalib.1106444

Received: May 18, 2020

Accepted: July 7, 2020

Published: July 10, 2020

Copyright $\odot 2020$ by author(s) and Open Access Library Inc.

This work is licensed under the Creative Commons Attribution International License (CC BY 4.0).

http://creativecommons.org/licenses/by/4.0/

\begin{abstract}
The article discusses the characteristics of the fractality of heart rate variability represented by a time series. An algorithm for calculating the Hurst exponent is presented, which allows one to evaluate the degree of chaotization of the signal in question. The capabilities of various multifractal analysis programs are shown, which require the use of different mathematical models.
\end{abstract}

\section{Subject Areas}

Computational Physics

\section{Keywords}

Multifractal, Herst Indicator, Heart Rate, Deterministic Chaos, Software

\section{Introduction}

Features of the cardiovascular system based on the study of heart rhythm allow you to obtain characteristics using mathematical processing, which are very significant for diagnostic and prognostic purposes. A significant limitation for the application of classical processing methods is the requirement of stationarity of the analyzed processes. Therefore, it becomes necessary to apply specialized approaches to the analysis of the cardiovascular system to adequately describe the processes occurring in this system, taking into account such significant factors as the essentially non-linear nature of the relationship between different indicators and between readings of one biological signal, significant variability of states, etc. Among these approaches, methods of nonlinear dynamics, the theory of dynamic chaos, fractal analysis, analysis of interval statistics, the theory of statistical solutions and some others can be distinguished [1].

Typically, in assessing the variability and degree of complexity of a heart rhythm, 
parametric statistics and spectral analysis are used. At the same time, it was found that the main share in the power spectrum of heart rate variability falls on chaotic components of a fractal nature. Therefore, the characteristics of fractality of the heart rhythm are increasingly being studied. Applying the methods of nonlinear dynamics to the analysis of ECG recording duration, you can get a more complete picture of complex changes in the regulation of the heart [2].

To assess the randomness of the heart rhythm, one can use such characteristics of fractal sets as the Hausdorff fractal dimension, correlation and informational dimension, Hurst exponent, generalized dimension and dimension of phase space [3] [4]. An approach is possible in which a number of times of shifts of the time series of the heart rhythm are considered as a homogeneous fractal (monofractal); however, one must consider the set described by this series as an inhomogeneous fractal process (multifractal). The term "heterogeneous" is understood as an uneven distribution of the points of a set over a fractal. The reason for the heterogeneity is the different probabilities of filling geometrically identical fractal elements, or in the general case, the mismatch of the probabilities of filling the geometrical size of the corresponding regions.

\section{Calculation Method}

One of the main fractal methods is $R / S$ analysis, which is a combination of statistical techniques and methods of analyzing time series (digital signals) that allow you to determine some of their important characteristics, such as the presence of non-periodic cycles, "temporary memory", etc. One of the fundamental $R / S$ analysis in the theory of fractals and deterministic chaos is the Hurst normalized swing method. The main parameter that can be found by the method of normalized magnitude is the Hurst exponent. Determining the value of this parameter allows one to judge the degree of randomization of the system as a whole, and the presence of the fractal nature of the signal under study in particular.

To calculate the Hurst exponent, the average voltage value $\langle U\rangle_{N}$ for $N$ time samples is found:

$$
\langle U\rangle_{N}=\frac{1}{N} \sum_{n=1}^{N} U(n)
$$

During the experiment, the average values are found for each time lag and these values are summarized in a table.

Next, the expression $X(n, N)$ is calculated, which represents the accumulated deviation of $U(n)$ from its average value $\langle U\rangle_{N}$, which is determined by a sum of the form

$$
X(n, N)=\sum_{p=1}^{n}\left\{U(p)-\langle U\rangle_{N}\right\}
$$

wherein the average value $\langle U\rangle_{N}$ is defined by Formula (1).

According to the method of normalized Hurst range, the range of deviations is determined through the minimum and maximum values of the accumulated 
deviation $X(n, N)$ :

$$
R(N)=X(n, N)-X(n, N)
$$

The standard deviation $S(n)$ can be calculated using the square root of the variance formula:

$$
S(N)=\sqrt{\frac{1}{\frac{1}{N}}} \sum_{m=1}^{n}\left\{U(n)-\langle U\rangle_{N}\right\}^{2}
$$

For most time series, the observed normalized $R / S$ range is described by the empirical relation and using expressions (1) and (2) has the form:

$$
R / S=(\alpha N)^{H}
$$

where $H$-Hurst exponent is an arbitrary constant. It should be noted that the span is called normalized, since it must be divided by the square root of the variance.

According to the theory of fractals, if the obtained value of the Hurst exponent is $H<0.5$, then the series under study has a "short-term" memory, i.e. is anti-persistent. Consequently, recent events in the system that generated it have a much greater influence on the further behavior of the system itself than earlier events. If $H>0.5$, then the time series is persistent and has a fractal structure. At $H=0.5$, the signal is stochastic noise and does not contain useful information.

\section{Experimental Study}

When processing the obtained data, the Hurst exponent is found by calculating the slope of the straight line approximating the dependence of the normalized range $R / S$ as a function of the delay $N$ in the double logarithmic scale along both axes of the coordinate system. The approximating line is obtained using the least squares method. A feature of the calculation is that the logarithms on both axes are selected on the basis of 10 , the value $\alpha$ is chosen equal to unity.

For one of the experiments, the dependence of the $R / S$ ratio on the delay $N$ is shown together with a straight line approximating it and is shown in Figure 1.

In Figure 1 the curves are identified with letters "a" and "b". "a" represents a Hurst exponent function for the examined signal and " $b$ " indicates an approximating curve.

The slope coefficient of the approximating straight line for the studied signal (i.e. the Hurst exponent) is $H>0.5$, which indicates its fractal nature. Thus, it can be argued that the process that generated this signal has a long-term memory, i.e. is persistent.

Figure 2 shows the change in the Hurst exponent depending on the interval number $n$. The information provided includes dimensionless quantities. The entire cardio cycle is divided into intervals between $R$ signals and the amount of those intervals is indicated with " $n$ " sign. The relationship of $R / S$ is a dimensionless 


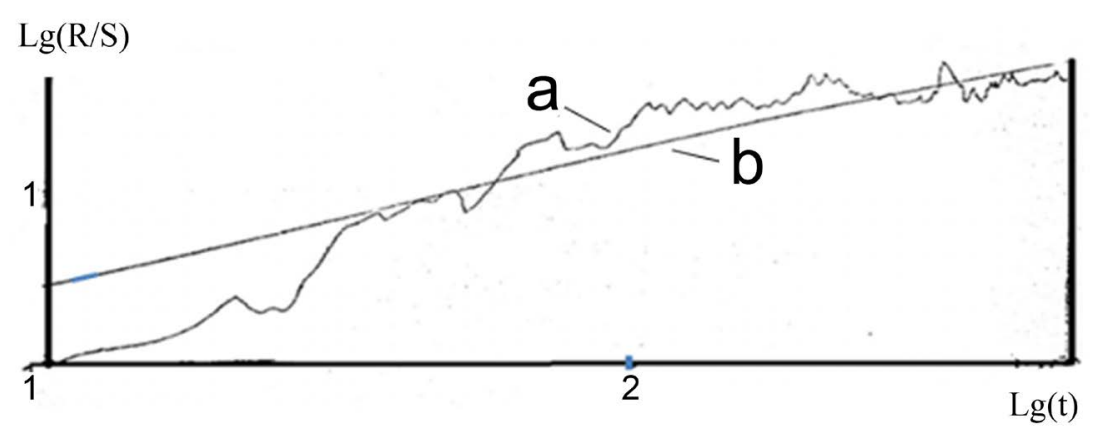

Figure 1. Change in the Hurst indicator on the time lag.

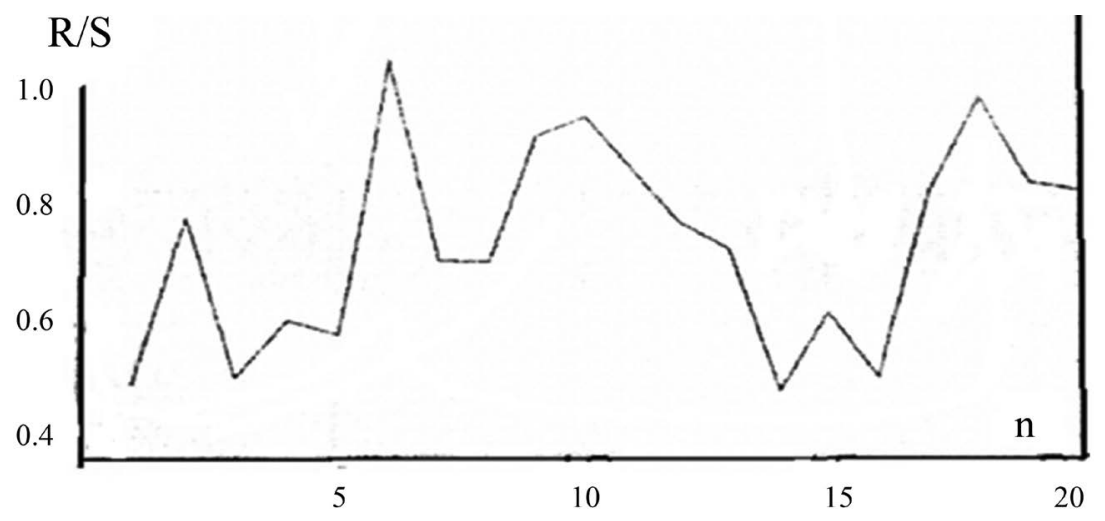

Figure 2. Change in the Hurst exponent depending on the interval number $n$.

quantity as well.

From Figure 2 we can conclude that the signal under study is fractal in nature. Therefore, it can be argued that the process that generated this signal has a long-term memory, i.e. is persistent.

For the data obtained in the experiment, programs for multifractal analysis are used [5].

The multifractal analysis program running under the Linux operating system is designed to obtain the scaling exponent and the spectrum of fractal dimensions. The Fractan program outputs several parameters, but the main for multifractal analysis is the Hurst parameter $(H)$, which characterizes the degree of indentation of the studied graph. Using the Multifrac program, you can get both the fractal dimension and the Hurst exponent. The application allows you to calculate the indicators of the signal under study, and visualize the data and display information in the appropriate fields. The Multifrac program allows you to calculate and display data without using mathematical models. The Multifral program allows you to determine a larger number of parameters compared to the Fractan program and multifractal analysis under Linux. This makes it possible to more accurately describe the characteristics of the processed signal.

\section{Conclusions}

- For a specific experimental implementation of heart rate variability, a multifractal signal analysis was carried out to determine the process randomness 
system.

- The dependences of the Hurst index on the time lag and depending on the interval number are given.

- The possibility of using three standard programs proposed by other researchers/scientists is presented.

- We proposed our own method for calculating chaotic signal parameters.

\section{Acknowledgements}

Great appreciation and immeasurable gratitude for the conducted studies that are a part of the scientific and technical work carried out at the Department of Biomedical Engineering of the Azerbaijan Technical University.

\section{Conflicts of Interest}

The authors declare no conflicts of interest regarding the publication of this paper.

\section{References}

[1] Bogachev, M.I., Kayumov, A.R., Krasichkov, A.S. and Markelov, O.A. (2012) Mathematical Methods for Identifying Regulatory and Statistical Patterns in Large Volume Biomedical and Economic Data. SPbETU “LETI”, Saint Petersburg, 176 p.

[2] Nemirko, A.P., Manilo, L.A. and Kalinichenko, A.N. (2017) Mathematical Analysis of Biomedical Signals and Data. Physmatlit, Moscow, $248 \mathrm{p}$.

[3] Cronover, R.M. (2006) Fractals and Chaos in Dynamical Systems. Postmarket Publ., Moscow, $488 \mathrm{p}$.

[4] Feder, E. (2013) Fractals. Springer Science + Business Media, LLC, Berlin, 282 p.

[5] Vinogradova, A.A. and Trutenko, S.V. (2011) Comparative Features of Multifractal Analysis Programs. News Universities “Instrument Making”, 54, 35-38.

https://doi.org/10.1016/j.dza.2011.04.022 